\title{
Search for point-like sources in the astrophysical muon neutrino flux with IceCube
}

\author{
The IceCube Collaboration \\ $\dagger$ http://icecube.wisc.edu/collaboration/authors/icrc17_icecube \\ E-mail: reimann@physik.rwth-aachen.de
}

The IceCube Collaboration has observed a high-energy diffuse astrophysical neutrino flux consistently in two detection channels: first using high-energy events interacting inside the detector and secondly with through-going muon-neutrino induced muons. In order to identify the sources of this flux, these muon-neutrino events are ideal messenger particles because of their excellent angular resolution. Here we present a search for point-like neutrino sources based on the same sample that was used to measure the diffuse high-energy astrophysical muon-neutrino flux with a live-time of six years. This high purity sample is restricted to the Northern hemisphere and consists of $\sim 340000$ events with a median angular resolution of $\sim 1^{\circ}$ at $1 \mathrm{TeV}$ that decreases to $\sim 0.3^{\circ}$ at $1 \mathrm{PeV}$. In this analysis an unbinned likelihood maximization is used that is optimized for point-like neutrino emission with the same characteristics as the diffuse muon neutrino flux. The sensitivity improves by $\sim 20 \%$ compared to previous analyses and is at a level of $E^{2} \partial \phi / \partial E=4 \cdot 10^{-13} \mathrm{TeV} \mathrm{cm}^{-2} \mathrm{~s}^{-1}$. No significant source was found in an unbiased sky scan on the Northern hemisphere and in a search based on a standard candidate source list of IceCube and ANTARES.

Corresponding author: R. Reimann*1

${ }^{1}$ III. Physikalisches Institut, RWTH Aachen University, D-52056 Aachen, Germany

35th International Cosmic Ray Conference - ICRC2017

10-20 July, 2017

Bexco, Busan, Korea

${ }^{*}$ Speaker. 


\section{Introduction}

IceCube is a cubic-kilometer neutrino detector with a total of 5160 optical sensors installed in the ice on 86 cable strings at the geographic South Pole [1,2] between depths of $1450 \mathrm{~m}$ and $2450 \mathrm{~m}$. Detector construction finished in 2010. Neutrino reconstruction relies on the optical detection of Cherenkov radiation emitted by secondary particles produced in neutrino interactions in the surrounding ice or the nearby bedrock. During construction, partial detector configurations were taking data with 59 strings from May 2009, and 79 strings from May 2010 on before IceCube became fully operational in May 2011.

In 2013 the IceCube Collaboration reported the observation of a diffuse astrophysical highenergy all-flavor neutrino flux using a sample of starting events $[3,4]$. This observation was confirmed by the measurement of a diffuse astrophysical high-energy muon-neutrino flux using the complementary detection channel of through-going muon-neutrinos with interaction vertex in the vicinity of the detector $[5,6,7]$. Those analyses are restricted to the Northern hemisphere to suppress the background of atmospheric muons that arises from cosmic ray air showers. In [6], the diffuse astrophysical high-energy neutrino flux was fitted by a power law with a spectral index of $2.13 \pm 0.13$ and a flux normalization of $0.90_{-0.27}^{+0.3} \times 10^{-18} \mathrm{GeV}^{-1} \mathrm{~cm}^{-2} \mathrm{~s}^{-1} \mathrm{sr}^{-1}$ at $1 \mathrm{TeV}$ neutrino energy. Until now, no significant source of these high-energy neutrinos could be identified e.g. see [8].

Track-like events from through-going muons are ideal to search for point-like sources. The background for this search are atmospheric neutrinos as well as uncorrelated diffuse astrophysical neutrinos. Atmospheric neutrinos can be distinguished from astrophysical neutrinos on a statistical level, because astrophysical neutrinos are expected to follow a much harder power law than atmospheric neutrinos with $\sim E^{-3.7}$.

Here, we present a scan for point-like sources on the Northern hemisphere down to $-5^{\circ}$ declination as well as a search based on a list of known candidate objects for the production of highenergy neutrinos. We use a sample of through-going muon neutrinos from 6 years of IceCube data [6] with about 340000 events from the Northern hemisphere with a known portion of astrophysical events. Despite of smaller live-time, the sensitivity increases by about $20 \%$ compared to [8].

\section{Data Sample}

As the data were taken with different partial configurations of IceCube the details of the event selections are different for different seasons. The event selection technique applied here are described in detail in [6]. At final selection level the muon neutrino purity is $>99.7 \%$ where the dominant part is atmospheric muon neutrinos from cosmic ray air showers [6]. These atmospheric neutrinos are an irreducible background and can just be separated on a statistical bases.

In total data with a live-time of 2032 days are analyzed containing about 340000 events. The event sample for the season from May 2011 to May 2012 has an overlap of about $80 \%$ with the selection presented in [8] for the same time range.

At high energies the muon direction is well correlated with the muon-neutrino direction $(<$ $0.1^{\circ}$ above $10 \mathrm{TeV}$ ) and the muon is reconstructed with a median angular uncertainty of about $0.5^{\circ}$ at $10 \mathrm{TeV}$. All events have been reconstructed with an improved reconstruction based on the 


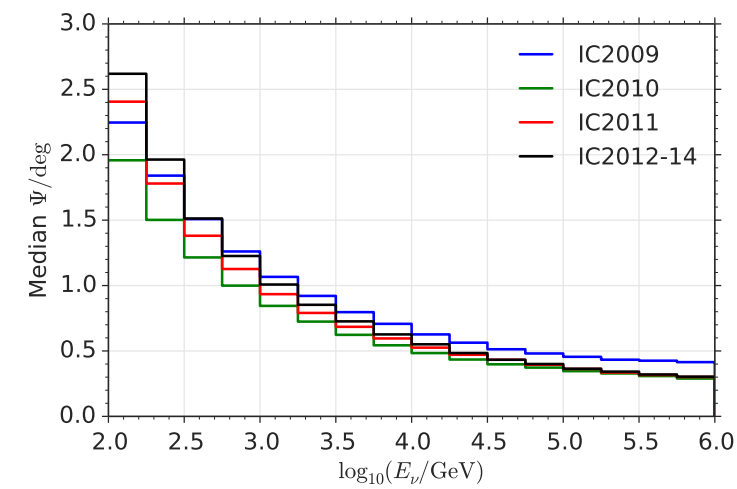

Figure 1: Median angular resolution vs $\log _{10}$ of neutrino energy for the different sub-selections labeled by the year.

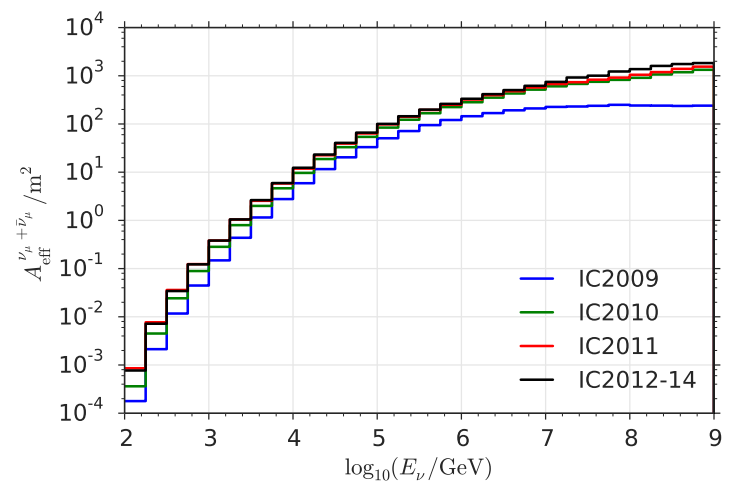

Figure 2: Effective area for neutrinos and antineutrinos from the Northern hemisphere vs $\log _{10}$ of neutrino energy for different sub-selections labeled by the year.

reconstruction techniques described in $[9,10]$. The median angular resolution $\Psi$ improves by about $10 \%$ compared to the reconstruction used in [8]. The median angular resolution for the different sub-selection labeled by the year of the selection are shown in Fig.1. The effective area for muon neutrino and muon anti-neutrinos for events from the Northern hemisphere of the different subselections is shown in Fig.2.

\section{Unbinned Likelihood Method}

The data sample is tested for clustering with an unbinned likelihood method as described in [11]. The likelihood function is given by

$$
\mathscr{L}=\prod_{j} \prod_{i \in j}\left[\frac{n_{s} \cdot w_{j}(\gamma)}{N_{j}} S^{\text {spat }}\left(\vec{x}_{i}, \vec{x}_{s}, \sigma_{i}\right) S_{j}^{\text {ener }}\left(E_{i}, \gamma\right)+\left(1-\frac{n_{s} \cdot w_{j}(\gamma)}{N_{j}}\right) B_{j}^{\text {spat }}\left(\vec{x}_{i}\right) B_{j}^{\text {ener }}\left(E_{i}\right)\right] \cdot P\left(\gamma \mid \gamma_{\text {astro }}\right)
$$

where $i$ is running over all events within a sub-sample $j . \vec{x}_{i}=\left(\alpha_{i}, \delta_{i}\right)$ is the reconstructed right ascension and declination, $E_{i}$ is the reconstructed energy and $\sigma_{i}$ is the estimated angular uncertainty on the reconstruction of event $i . N_{j}$ is the total sample size of sub-sample $j . n_{s}$ is the number of signal events from the assumed source position at $\vec{x}_{s}$ with a spectral index of $\gamma$. The signal and background probability is factorized in a spatial and an energy part. The spatial background probability $B_{j}^{\text {spat }}$ is uniform in right ascension due to IceCube's special position at the South Pole. The declination dependence of the spatial background PDF $B_{j}^{\text {spat }}$ and the energy background PDF $B_{j}^{\text {ener }}$ is estimated using Monte Carlo simulation weighted to the best fit parametrization obtained from [6] including the diffuse atmospheric and astrophysical components, specializing the PDFs for this sample. This allows a better extrapolation to sparse populated regions in the energy, declination plane than using experimental data only. Also the energy signal PDF $S_{j}^{\text {ener }}$ and $w_{j}$ are estimated using Monte Carlo simulation assuming a power law spectrum of the source with spectral index $\gamma$. $w_{j}$ is the fraction of signal events that are expected from a specific sub-sample $j$ that is calculated using the exposure and effective area of the sub-samples. The spatial signal probability $S^{\text {spat }}$ is assumed to be Gaussian with an event individual uncertainty of $\sigma_{i}$. 
To focus on those sources that produce the observed spectrum of astrophysical events, we add a Gaussian prior for the spectral index $P\left(\gamma \mid \gamma_{a s t r o}=2.13 \pm 0.13\right)$. The position and the width of the prior are taken from the best fit of [6]. As the source spectrum is not strongly constrained by the few events that contribute to the source the prior has the largest handle on the fit of $\gamma$ and thus the spectral index is effectively fixed allowing just for a small variation.

For a potential source position, the likelihood is maximized with respect to $n_{s}$ and $\gamma$ with $n_{s} \geq 0$ and a likelihood ratio test is performed to compare the best fit likelihood to the null hypothesis of no significant clustering with $n_{s}=0$. The ratio is the test statistic

$$
T S=-2 \cdot \log \left[\frac{\mathscr{L}\left(\vec{x}_{s}, n_{s}=0\right)}{\mathscr{L}\left(\vec{x}_{s}, \hat{n}_{s}, \hat{\gamma}\right)}\right] \quad n_{s}>0
$$

for $n_{s}>0$ with best fit values $\hat{n}_{s}$ and $\hat{\gamma}$. Up to $80 \%$ of background trials accumulate in a peak at $\hat{n}_{s}=0$ and the sensitivity would be under-estimated if these fits get the same $T S$-value. Thus we expand the shape of the $\log \mathscr{L}$ function at $n_{s}=0$ with a Taylor-expansion of second order to estimate the minimum $T S$ value for negative $n_{s}$ values resolving the peak at $T S=0$.

Due to IceCube's distinguished position at the South Pole, background samples can be generated by scrambling experimental data uniformly in right ascension. It was found that the $T S$ is slightly declination dependent. Thus the background $T S$ is generated using background trials for many different declinations. These $T S$-distributions are used to calculate a pre-trial p-value.

The sensitivity and discovery potential on the source flux normalization assuming an $E^{-2}$ power law flux is shown in Fig.5. The sensitivity corresponds to a 90\% C.L. averaged upper limit and the discovery potential gives the median source flux resulting in a $5 \sigma$ discovery. The sensitivity and discovery potential are declination dependent. For comparison the sensitivity and discovery potential from [8] are shown. Despite of one year shorter live-time, this analysis outperforms the analysis in [8] by about $10 \%-20 \%$. This is caused by multiple reasons: 1 . the use of an improved angular reconstruction, 2. a slightly better optimized event selection near the horizon, 3 . the use of background PDFs in the likelihood that are optimized on the parametrization from [6] which improves sensitivity especially for higher energies, 4 . the fact that due to the prior on the spectral index the number of source hypotheses are reduced which results in a steeper falling $T S$ and 5. the use of negative $T S$ values which allows not to under-estimate the sensitivity especially near the pole ( $\sin \delta \sim 1$ ) where the background changes strongly. As this search is optimized for hard-spectrum sources it does not outperform the analysis in [8] for soft-spectrum sources, e.g. $E^{-3}$.

\subsection{Unbiased Scan}

We perform an unbiased scan of the full Northern hemisphere going down to $-5^{\circ}$ declination. The scan is performed on a grid with a typical resolution of about $0.1^{\circ}$. For each grid point the pre-trial p-value is calculated. We select the most significant spot from the scan using the pre-trial p-value. As we test many points in the scan we have to correct for the fact that we choose the most significant point in our scan. Therefore, we repeat the procedure with scrambled background samples. By comparing the pre-trial p-values from the most significant points in the background sample to the experimental pre-trial p-value we can calculate the post-trial p-value. The background pre-trial p-value distribution of the most significant point can be described by

$$
p_{\text {pre-trial }} \cdot\left(1-p_{\text {pre-trial }}\right)^{N-1}
$$




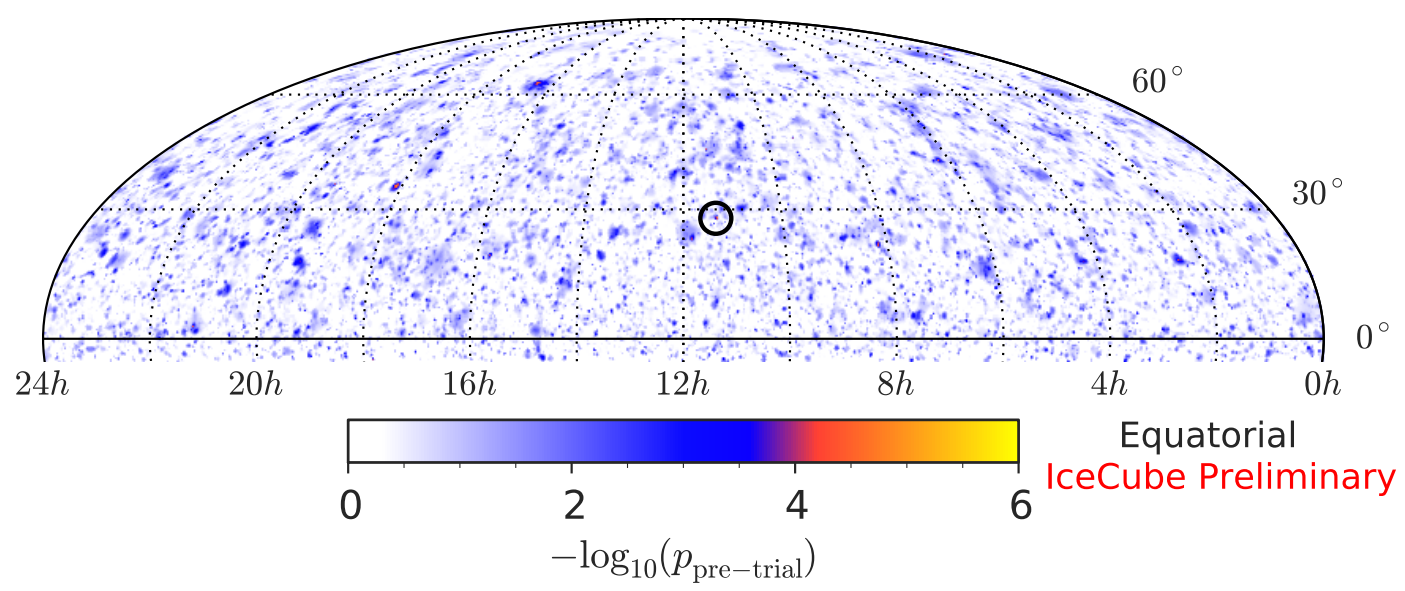

Figure 3: Pre-trial p-value sky map of the Northern hemisphere scan in equatorial coordinates down to $-5^{\circ}$ declination. The pre-trial p-value is given as $-\log _{10}\left(p_{\text {pre-trial }}\right)$. The position of the hottest spot is indicated by a black circle.

with an effective number of trials $N$ of about 130000 .

\subsection{A-Priori Source List}

Weak sources suffer from the large number of trials of about 130000 within the unbiased scan and may appear non-significant after trial correction. However a standard IceCube and ANTARES a-priori source list contains 34 promising candidates for high-energy neutrino emission in the Northern hemisphere [8], Tab. 1, reducing the trial factor to about 34. The sources were selected mainly due to their observation in gamma rays. They belong to various categories e.g. Galactic pulsar wind nebulae and supernovae remnants or extra-galactic BL Lacs and FSRQs. We test each source from this list individually. We select the most significant source and apply a trial-correction using background scrambled samples as done for the unbiased scan.

\section{Results}

No significant clustering was found above background expectation. Both the unbiased scan on the Northern hemisphere and the source list are compatible with background only.

The pre-trial p-value map of the unbiased scan is shown in Fig.3. The hottest spot in the scan is indicated by a black circle and is located at right ascension $\alpha=170.16^{\circ}$, declination $\delta=27.91^{\circ}$. The Galactic coordinates are $b_{\text {gal }}=69.88^{\circ}, l_{\text {gal }}=205.45^{\circ}$. The best fit signal strength is $n_{s}=9.88$ with a fitted spectral index of $\gamma=2.118$. The $T S$-value is 17.36 which corresponds to $p_{\text {pre-trial }}=$ $10^{-5.14}$. The post-trial corrected p-value is $90.5 \%$ and is thus compatible with background only. A zoom into the pre-trial p-value landscape around the hottest spot position is shown in Fig.4. In addition positions for sources from the Fermi 2FGL, Fermi 3FGL and Fermi 3FHL catalogs are shown $[12,13,14]$. The closest of these sources is 2 degree away.

The $\mathscr{L}$ fit results for each source of the source list are shown in Tab.1. Sources are sorted by p-value and the best fit $n_{s}$ as well as the $T S$ and the equatorial coordinates are given. The 


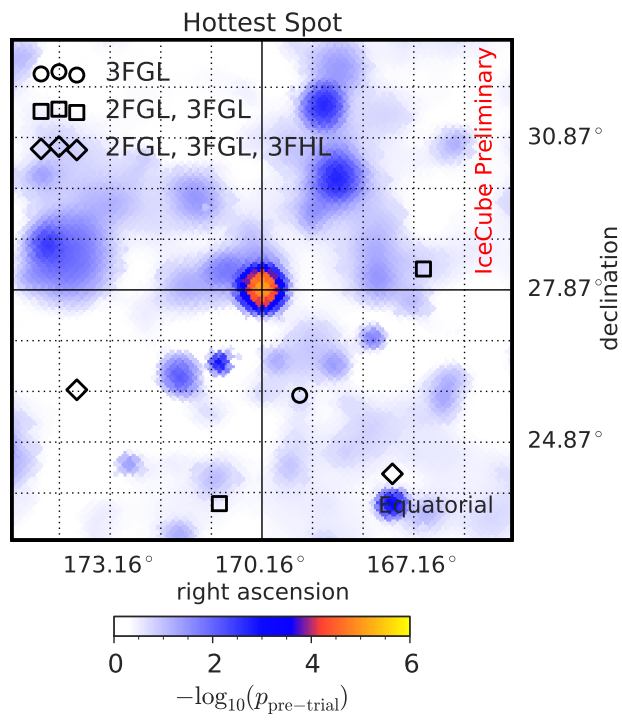

Figure 4: Pre-trial p-value landscape centered on the position of hottest spot of the unbiased scan in equatorial coordinates. The pre-trial p-value is given as $-\log _{10}\left(p_{\text {pre-trial }}\right)$. In addition sources of Fermi 2FGL, 3FGL and 3 FHL catalog are indicated by markers $[12,13,14]$.

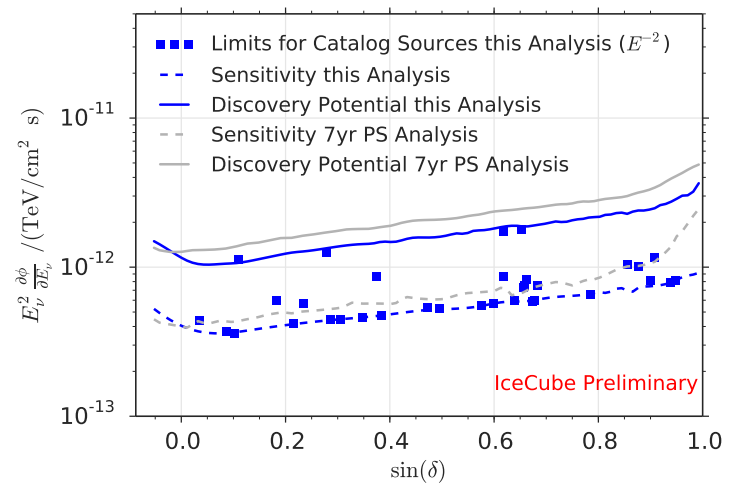

Figure 5: Upper limits on the flux normalization at $90 \%$ C.L. vs sine of declination of there source in the source list. In addition the sensitivity (dashed) and discovery potential (solid) flux for this analysis (blue) and from [8] (gray) are shown assuming an $E^{-2}$ source spectrum.

most significant source in the a-priori source list is MGRO J1908+06 with a pre-trial p-value of $0.32 \%$. Comparing to the pre-trial p-value distribution of the most significant source from scrambled background samples the post-trial p-value is $10.2 \%$, which is compatible with background. As no significant source was found, we calculate the $90 \%$ C.L. Neyman upper limit [15] on the flux normalization $\Phi_{0}$ for $v_{\mu}+\bar{v}_{\mu}$ for each source. The upper limits on the flux normalization are listed in Tab. 1 and are shown as blue squares in Fig. 5 vs the sine of the declination of the source together with the sensitivity. Note that in case of under-fluctuations the limit was set to the sensitivity level of the analysis. Also note $90 \%$ upper limits can exceed the discovery potential as long as the best fit flux is below.

From Table 1 it can be seen that we find four different sources with a pre-trial p-value below $1 \%$. In an a posteriori test we have quantified the probability to find such an excess of several small p-values. For this, we have calculated the binomial probability

$$
P_{\text {binom }}(k)=\left(\begin{array}{c}
N \\
k
\end{array}\right) p_{\mathrm{k}}^{k}\left(1-p_{\mathrm{k}}\right)^{N-k}
$$

to find $k$ out of $N=34$ sources with a pre-trial p-value smaller then $p_{\mathrm{k}}$. Here $p_{\mathrm{k}}$ is the pre-trial p-value of source $k$, if sources are sorted by their pre-trial p-value. The maximum significance with almost $3.8 \sigma$ pre-trial is found for $k=4$. Again, we have to correct for the fact that we choose $k$ which results in the maximal significance. Thus we have repeated this procedure on scrambled background samples, each time selecting the most significant excess of p-values. After trial correction the $\mathrm{p}$-value to find four sources with a pre-trial $\mathrm{p}$-value $<0.72 \%$ is $0.3 \%$ reducing the significance to $2.75 \sigma$. 


\begin{tabular}{|c|c|c|c|c|c|c|c|}
\hline Source & Type & $\alpha[\operatorname{deg}]$ & $\delta[\operatorname{deg}]$ & $\mathrm{p}$-value & $T S$ & $n_{s}$ & $\Phi_{0}\left[\mathrm{TeV} \mathrm{cm}^{-2} \mathrm{~s}^{-1}\right]$ \\
\hline MGRO J1908+06 & $\mathrm{NI}$ & 286.99 & 6.27 & 0.0032 & 6.284 & 3.28 & $1.13 \cdot 10^{-12}$ \\
\hline Cyg A & SRG & 299.87 & 40.73 & 0.0049 & 6.335 & 4.30 & $1.78 \cdot 10^{-12}$ \\
\hline 4C 38.41 & FSRQ & 248.81 & 38.13 & 0.0055 & 5.686 & 6.62 & $1.72 \cdot 10^{-12}$ \\
\hline $3 \mathrm{C} 454.3$ & FSRQ & 343.50 & 16.15 & 0.0072 & 5.503 & 5.98 & $1.26 \cdot 10^{-12}$ \\
\hline Crab Nebula & PWN & 83.63 & 22.01 & 0.1188 & 0.709 & 4.32 & $8.65 \cdot 10^{-13}$ \\
\hline Cas A & SNR & 350.85 & 58.81 & 0.2069 & 0.033 & 0.88 & $1.05 \cdot 10^{-12}$ \\
\hline 1ES $1959+650$ & BL Lac & 300.00 & 65.15 & 0.2069 & 0.124 & 1.69 & $1.17 \cdot 10^{-12}$ \\
\hline PKS $1502+106$ & FSRQ & 226.10 & 10.52 & 0.2322 & -0.000 & 0.00 & $5.98 \cdot 10^{-13}$ \\
\hline Mrk 421 & BL Lac & 166.11 & 38.21 & 0.2433 & 0.029 & 0.48 & $8.68 \cdot 10^{-13}$ \\
\hline NGC 1275 & SRG & 49.95 & 41.51 & 0.2582 & 0.007 & 0.25 & $8.31 \cdot 10^{-13}$ \\
\hline LSI 303 & $\mathrm{XB} / \mathrm{mqso}$ & 40.13 & 61.23 & 0.2843 & 0.001 & 0.17 & $1.01 \cdot 10^{-12}$ \\
\hline PKS 0528+134 & FSRQ & 82.73 & 13.53 & 0.2870 & -0.002 & 0.00 & $5.74 \cdot 10^{-13}$ \\
\hline Cyg OB2 & SFR & 308.09 & 41.23 & 0.3174 & -0.002 & 0.00 & $7.53 \cdot 10^{-13}$ \\
\hline Cyg X-3 & $\mathrm{XB} / \mathrm{mqso}$ & 308.11 & 40.96 & 0.3230 & -0.003 & 0.00 & $7.28 \cdot 10^{-13}$ \\
\hline $3 \mathrm{C} 66 \mathrm{~A}$ & BL Lac & 35.67 & 43.04 & 0.3306 & -0.001 & 0.00 & $7.50 \cdot 10^{-13}$ \\
\hline $3 \mathrm{C} 273$ & FSRQ & 187.28 & 2.05 & 0.3807 & -0.014 & 0.00 & $4.42 \cdot 10^{-13}$ \\
\hline W Comae & BL Lac & 185.38 & 28.23 & 0.4420 & -0.055 & 0.00 & $5.37 \cdot 10^{-13}$ \\
\hline TYCHO & SNR & 6.36 & 64.18 & 0.4471 & -0.019 & 0.00 & $8.14 \cdot 10^{-13}$ \\
\hline 1ES $0229+200$ & BL Lac & 38.20 & 20.29 & 0.4762 & -0.059 & 0.00 & $4.47 \cdot 10^{-13}$ \\
\hline BL Lac & BL Lac & 330.68 & 42.28 & 0.5104 & -0.028 & 0.00 & $5.58 \cdot 10^{-13}$ \\
\hline Cyg X-1 & $\mathrm{XB} / \mathrm{mqso}$ & 299.59 & 35.20 & 0.5422 & -0.106 & 0.00 & $4.93 \cdot 10^{-13}$ \\
\hline M87 & SRG & 187.71 & 12.39 & 0.6711 & -0.256 & 0.00 & $2.85 \cdot 10^{-13}$ \\
\hline Mrk 501 & BL Lac & 253.47 & 39.76 & 0.6847 & -0.172 & 0.00 & $3.51 \cdot 10^{-13}$ \\
\hline S5 0716+71 & BL Lac & 110.47 & 71.34 & 0.7230 & -0.380 & 0.00 & $3.84 \cdot 10^{-13}$ \\
\hline PKS 0235+164 & BL Lac & 39.66 & 16.62 & 0.7355 & -0.400 & 0.00 & $2.04 \cdot 10^{-13}$ \\
\hline H $1426+428$ & BL Lac & 217.14 & 42.67 & 0.7890 & -0.243 & 0.00 & $1.96 \cdot 10^{-13}$ \\
\hline IC443 & SNR & 94.18 & 22.53 & 0.8153 & -0.457 & 0.00 & $1.22 \cdot 10^{-13}$ \\
\hline HESS J0632+057 & $\mathrm{XB} / \mathrm{mqso}$ & 98.24 & 5.81 & 0.8359 & -0.917 & 0.00 & $1.01 \cdot 10^{-13}$ \\
\hline SS433 & $\mathrm{XB} / \mathrm{mqso}$ & 287.96 & 4.98 & 0.8738 & -1.085 & 0.00 & $1.01 \cdot 10^{-13}$ \\
\hline M82 & SRG & 148.97 & 69.68 & 0.8887 & -0.888 & 0.00 & $1.83 \cdot 10^{-13}$ \\
\hline 3C 123.0 & SRG & 69.27 & 29.67 & 0.9055 & -0.747 & 0.00 & $1.30 \cdot 10^{-13}$ \\
\hline 1ES $2344+514$ & BL Lac & 356.77 & 51.70 & 0.9264 & -0.808 & 0.00 & $1.58 \cdot 10^{-13}$ \\
\hline Geminga & PWN & 98.48 & 17.77 & 0.9754 & -2.424 & 0.00 & $1.16 \cdot 10^{-13}$ \\
\hline MGRO J2019+37 & PWN & 305.22 & 36.83 & 0.9884 & -3.191 & 0.00 & $1.39 \cdot 10^{-13}$ \\
\hline \multicolumn{8}{|c|}{ Hottest spot of the unbiased scan on the Northern hemisphere } \\
\hline- & - & 170.16 & 27.87 & $10^{-5.14}$ & 17.271 & 10.28 & - \\
\hline
\end{tabular}

Table 1: Results of the a priori defined source list search. The fitted spectral index $\gamma$ is not given as it is effectively fixed by the introduced prior. The most significant source is MGRO J1908+06 with a post-trial p-value of $10.2 \%$. In addition the fit values of the hottest spot of the unbiased sky scan is given.

The four sources are MGRO J1908+06, Cyg A, 3C454.3 and 4C 38.41 that are three extragalactic and one Galactic source. The type of MGRO J1908+06 is not identified, Cyg A is a FR-II radio galaxy and 3C454.3 and 4C 38.41 are both FSRQ. We note, that we have not found a physical reason to combine these sources belonging to different source classes and that the test for a population of small p-values has been introduced a posteriori. 


\section{Conclusions}

We have analyzed 6 years of IceCube data for a clustering of through-going muon neutrinos using an improved unbinned likelihood method. No significant source was found in a scan of the Northern hemisphere. Also, the search for significant neutrino emission from objects on a predefined list of candidates results in a post-trial p-value of $10.2 \%$, compatible with background. The object on the list with the smallest post-trial p-value is MGRO J1908+06.

With the end of the data taking season 2016 in May 2017, this analysis can be extended to two more years of data. In addition to the analysis presented here, we will also perform a test for a population of sub-threshold sources, similar to the one described in [8]. Currently the event selection is re-optimized to take advantage from the features of the event selection described in [6] and [8].

\section{References}

[1] IceCube Collaboration, A. Achterberg et al., Astropart. Phys. 26 (2006) 155 - 173.

[2] IceCube Collaboration, M. Aartsen et al., JINST 12 (2017) P03012.

[3] IceCube Collaboration, M. G. Aartsen et al., Science 342 (2013).

[4] IceCube Collaboration, PoS (ICRC2017) 981 (these proceedings).

[5] IceCube Collaboration, M. G. Aartsen et al., Phys. Rev. Lett. 115 (2015) 081102.

[6] IceCube Collaboration, M. G. Aartsen et al., Astrophys. J. 833 (2016) 3.

[7] IceCube Collaboration, PoS ( ICRC2017) 1005 (these proceedings).

[8] IceCube Collaboration, M. G. Aartsen et al., Astrophys. J. 835 (2017) 151.

[9] AMANDA Collaboration, J. Ahrens et al., Nucl. Instrum. Meth. A 524 (2004) 169 - 194.

[10] K. Schatto. PhD thesis, Johannes Gutenberg-Universität, 2014.

[11] J. Braun et al., Astropart. Phys. 29 (2008) 299 - 305.

[12] Fermi-LAT Collaboration, P. L. Nolan et al., Astrophys. J. Suppl. S. 199 (2012) 31.

[13] Fermi-LAT Collaboration, F. Acero et al., Astrophys. J. Suppl. S. 218 (2015) 23.

[14] Fermi-LAT Collaboration, M. Ajello et al., arXiv:1702.00664.

[15] J. Neyman, Phil. Trans. R. Soc. A 236 (1937) 333-380. 